G

\title{
Autoionization Rates for Sodium- and Magnesium-Like Tungsten and Gold
}

\author{
G. GÜNDAY KONAN* AND L. ÖZDEMİR \\ Sakarya University, Physics Department, 54187, Sakarya, Turkey
}

(Received August 5, 2016; in final form June 7, 2017)

The autoionization process has an important role in many areas such as satellite line formation, inner-shell ionization, and diagnostics of astrophysical and fusion plasmas. We have here reported autoionization rates for some levels of Na-like tungsten $\left(\mathrm{W}^{63+}\right)$ and gold $\left(\mathrm{Au}^{68+}\right)$, and $\mathrm{Mg}$-like tungsten $\left(\mathrm{W}^{62+}\right)$ and gold $\left(\mathrm{Au}^{67+}\right)$ using AUTOSTRUCTURE atomic code. In calculations, the Breit interactions, quantum electrodynamics and configuration interaction effects have been considered. The results obtained for tungsten ions have been compared with other available results. Also new results for gold ions have been presented.

DOI: 10.12693/APhysPolA.132.1290

PACS/topics: 32.80.Zb, 31.15.A-

\section{Introduction}

Accurate autoionization data are necessary for the progress of spectroscopy due to observing in a large variety of atomic and molecular spectra, and also autoionization rates differ by orders of magnitude between states of the same electronic configuration [1]. In addition, the study of autoionizing states of an atom has been of great interest for such areas as plasma physics, astrophysics, ultracold atomic physics, surface science and chemistry (the Auger spectroscopy), laser isotope separation, development of new lasers, propagation of ultra-short laser pulses without absorption in an autoionizing media, laser without population inversion and the role of autoionizing states in coherent control [2-10]. Therefore, many theoretical and experimental efforts have been directed towards accurate calculation or measurements of autoionization data in various atoms and ions. Different experimental and theoretical methods in [11] have been used to study autoionization: cloud chamber (the Auger effect), continuous ultraviolet absorption spectroscopy and electron scattering methods as experimental, and the Feshbach projection operator formalism, multiconfiguration energy bound method, scattering close-coupling method, multichannel configuration interaction method, root-stabilization method, complex-coordinate method, truncated diagonalization method, many-body perturbation theory, time stability theory, and derivative method as theoretical.

Atomic data of highly ionized gold $(\mathrm{Au}, Z=79)$ ions are needed, in particular, in plasma science, fusion reaction, biomedical applications, high energy astrophysics, and other scientific research fields [12]. Also tungsten (W, $Z=74$ ) has become a center of focus of fusion research, being a main candidate for plasma-facing compo-

*corresponding author; e-mail: ggunday@sakarya.edu.tr nents [13]. Accurate autoionization data and dielectronic recombination which is the inverse process of autoionization, for a large range of charge states of gold and tungsten have been presented in the literature. Safronova et al. reported many articles about excitation energies, radiative and autoionization rates, dielectronic satellite lines, and dielectronic recombination rates for various tungsten ions [14-21]. Also dielectronic recombination of tungsten ions was presented [22-26]. Gold ions were less studied than tungsten ions. Dielectronic recombination of some gold ions was reported [27-30].

In this paper we have calculated autoionization rates for Na-like tungsten $\left(\mathrm{W}^{63+}\right)$ and gold $\left(\mathrm{Au}^{68+}\right)$, and $\mathrm{Mg}$ like tungsten $\left(\mathrm{W}^{62+}\right)$ and gold $\left(\mathrm{Au}^{67+}\right)$, using atomic structure code (AUTOSTRUCTURE) in the framework of the Breit-Pauli distorted wave approach developed by Badnell [31]. Na- and Mg-like ions which have only one or two valence electrons, have a relatively simple atomic system for atomic calculations. The results include the Breit interactions (magnetic interaction between the electrons and retardation effects of the electron-electron interaction), quantum electrodynamics (self-energy and vacuum polarization), and electron correlation effects in the framework of configuration interactions.

\section{Calculation method}

AUTOSTRUCTURE code [31-33] is a general program for the calculation of atomic and ionic energy levels, radiative and autoionization rates and photoionization cross-sections using non-relativistic or semirelativistic wave functions. It is based on SUPERSTRUCTURE [34]. In this code, the configuration set is chosen optionally and there is added new configuration to improve accuracy (a configuration interaction expansion, CI expansion). The CI expansion is related to the choice of radial functions. Each $(n l)$ radial function is calculated in the Thomas-Fermi or the Slater-type-orbital potential model. The Hamiltonian in any coupling model (LS, IC, or ICR) is diagonalized to obtain eigenvalues and 
eigenvectors with which to construct the rates. Detailed information on the method of this code can be found in [32-34].

All atoms or ions with two or more electron have autoionization states. The autoionization transition probability rate, $A_{a}$ is given in first-order perturbation theory by $[35]$ :

$$
A_{a}\left(t^{\prime} \rightarrow t\right)=\left(\frac{8 I_{H}}{\hbar}\right)\left|\left\langle t|H| t^{\prime}\right\rangle\right|^{2},
$$

where $I_{H} / \hbar=2.06707 \times 10^{16} \mathrm{~s}^{-1}$ and $t=C S L p, C$ denotes a configuration and $p$ the parity, which is conserved in $[32]$.

\section{Results and discussion}

In our previous works we reported energy levels and radiative parameters for tungsten and gold ions [36-39]. Now the autoionization rates which have played an important role in the progress of spectroscopy have been presented for Na-like tungsten $\left(\mathrm{W}^{63+}\right)$, Mg-like tungsten $\left(\mathrm{W}^{62+}\right)$, Na-like gold $\left(\mathrm{Au}^{68+}\right)$ and $\mathrm{Mg}$-like gold $\left(\mathrm{Au}^{67+}\right)$ using AUTOSTRUCTURE code [31]. Na-like ions and $\mathrm{Mg}$-like ions have the ground-state configuration of $1 s^{2} 2 s^{2} 2 p^{6} 3 s$ and $1 s^{2} 2 s^{2} 2 p^{6} 3 s^{2}$, respectively. We have here studied different configuration sets for each ion. These configuration sets are given in Table I.

TABLE I

Configurations considered for calculations.

\begin{tabular}{l|l}
\hline \hline & Configuration \\
\hline $\mathrm{Au}^{68+}$ & $2 p^{5} 3 s 3 p+2 p^{5} 3 s 3 d+2 p^{5} 3 p^{2}+2 p^{5} 3 p 3 d+2 p^{5} 3 d^{2}$ \\
$\mathrm{~W}^{63+}$ & $3 s+3 p+3 d+4 s+4 p+4 d+4 f+2 s 2 p^{6} 3 s 3 p+$ \\
& $2 p^{5} 3 s 3 d+2 p^{5} 3 p^{2}+2 p^{5} 3 p 3 d+2 p^{5} 3 p 4 p+2 p^{5} 3 p 4 d+$ \\
& $2 p^{5} 3 d^{2}+2 p^{5} 3 d 4 p+2 p^{5} 3 d 4 d+2 p^{5} 3 d 4 f$ \\
$\mathrm{Au}^{67+}$ & $3 s^{2}+3 s 3 p+3 s 3 d+3 s 4 s+3 s 4 p+3 s 4 d+3 s 4 f+$ \\
& $3 p^{2}+3 p 3 d+3 p 4 s+3 d^{2}+3 d 4 s+3 d 4 f+4 s 5 p+$ \\
& $4 s 5 d+4 s 5 f+4 s 5 g+4 s 6 d+4 p 4 f+4 p 5 p+4 p 5 g+$ \\
& $4 p 6 g+4 d^{2}+4 d 4 f+4 d 5 g+4 f^{2}+4 f 5 s+4 f 5 d+$ \\
& $4 f 5 f+4 f 6 d+4 f 6 f$ \\
$\mathrm{~W}^{62+}$ & $3 s^{2}+3 s 3 p+3 s 3 d+3 s 4 s+3 s 4 p+3 s 4 d+3 s 4 f+$ \\
& $3 s 5 s+3 s 5 p+3 p^{2}+3 p 3 d+3 p 4 s+3 p 4 p+$ \\
& $3 d^{2}+2 p^{5} 3 s^{2} 3 p+2 p^{5} 3 s 3 p^{2}+2 p^{5} 3 p^{3}+2 p^{5} 3 s^{2} 3 d+$ \\
& $2 p^{5} 3 s^{2} 4 s+2 p^{5} 3 s 3 p 3 d+2 p^{5} 3 p^{2} 3 d+2 p^{5} 3 d^{3}+$ \\
& $2 p^{5} 3 s 3 d^{2}+2 p^{5} 3 s 3 p 4 s+4 s^{2}+4 s 4 p+4 s 4 d+4 s 5 p+$ \\
& $4 s 5 d+4 s 5 f+4 s 5 g+4 s 6 d+4 p 4 f+4 p 5 p+4 p 5 g+$ \\
& $4 p 6 g+4 d^{2}+4 d 4 f+4 d 5 g+4 f^{2}+4 f 5 s+4 f 5 d+$ \\
& $4 f 5 f+4 f 6 d+4 f 6 f$
\end{tabular}

In our early works, we studied with different configuration sets for calculations according to valencevalence correlation and core-valence correlation, within the framework of configuration interaction expansion. Beside quantum electrodynamics (QED, self-energy and vacuum polarization) and the Breit interaction (magnetic interaction between the electrons and retardation effects of the electron-electron interaction) contributions were investigated for tungsten and gold ions in [36-39]. All these contributions and correlations effects play an essential role in atomic calculations.
TABLE II

Autoionization rates $\left(A_{a}\right.$, in $\left.\mathrm{s}^{-1}\right)$ for $\mathrm{Na}$ - and $\mathrm{Mg}$-like tungsten ions. Numbers in brackets represent powers of 10. The other works have been obtained from Ref. [14] for Na-like W ( $\left.\mathrm{W}^{63+}\right)$ and Ref. [15] for Mg-like W ( $\left.\mathrm{W}^{62+}\right)$.

\begin{tabular}{|c|c|c|c|}
\hline \multicolumn{4}{|c|}{ Na-like $\mathrm{W}\left(\mathrm{W}^{63+}\right)$} \\
\hline \multicolumn{2}{|c|}{ level } & this work & other works \\
\hline $2 p^{5} 3 d^{2}$ & ${ }^{2} F_{5 / 2}$ & $8.66(14)$ & $2.06(14)^{a}$ \\
\hline $2 p^{5} 3 p^{2}$ & ${ }^{2} P_{1 / 2}$ & $5.88(14)$ & $1.52(14)^{a}$ \\
\hline $2 p^{5} 3 p^{2}$ & ${ }^{2} P_{3 / 2}$ & $1.41(13)$ & $5.20(13)^{a}$ \\
\hline $2 p^{5} 3 s 3 d$ & ${ }^{4} D_{3 / 2}$ & $9.52(13)$ & $3.05(13)^{a}$ \\
\hline $2 p^{5} 3 d^{2}$ & ${ }^{4} G_{5 / 2}$ & $1.18(14)$ & $0.34(14)^{a}$ \\
\hline $2 p^{5} 3 d 4 d$ & ${ }^{4} G_{5 / 2}$ & $2.48(13)$ & $2.40(13)^{a}$ \\
\hline $2 p^{5} 3 d^{2}$ & ${ }^{2} D_{5 / 2}$ & $5.55(13)$ & $1.90(13)^{a}$ \\
\hline $2 p^{5} 3 d^{2}$ & ${ }^{2} F_{7 / 2}$ & $1.93(14)$ & $1.22(14)^{a}$ \\
\hline $2 p^{5} 3 p 4 p$ & ${ }^{2} P_{3 / 2}$ & $2.60(13)$ & $5.25(13)^{a}$ \\
\hline $2 p^{5} 3 d 4 d$ & ${ }^{2} F_{5 / 2}$ & $1.30(14)$ & $1.15(14)^{a}$ \\
\hline $2 p^{5} 3 d 4 d$ & ${ }^{2} F_{7 / 2}$ & $5.28(13)$ & $5.08(13)^{a}$ \\
\hline $2 p^{5} 3 p 3 d$ & ${ }^{2} D_{3 / 2}$ & $2.07(14)$ & $1.11(14)^{a}$ \\
\hline $2 p^{5} 3 p 3 d$ & ${ }^{4} F_{3 / 2}$ & $4.99(13)$ & $4.17(13)^{a}$ \\
\hline $2 p^{5} 3 p 4 d$ & ${ }^{2} D_{3 / 2}$ & $1.84(13)$ & $3.50(13)^{a}$ \\
\hline $2 p^{5} 3 p 3 d$ & ${ }^{2} P_{3 / 2}$ & $6.44(13)$ & $3.92(13)^{a}$ \\
\hline $2 p^{5} 3 p 3 d$ & ${ }^{2} D_{5 / 2}$ & $8.97(13)$ & $9.05(13)^{a}$ \\
\hline $2 p^{5} 3 p 4 d$ & ${ }^{2} D_{5 / 2}$ & $2.64(13)$ & $2.46(13)^{a}$ \\
\hline $2 p^{5} 3 d 4 p$ & ${ }^{2} D_{3 / 2}$ & $2.39(13)$ & $8.55(13)^{a}$ \\
\hline $2 p^{5} 3 d 4 p$ & ${ }^{2} D_{5 / 2}$ & $2.99(13)$ & $3.68(13)^{a}$ \\
\hline $2 p^{5} 3 d 4 f$ & ${ }^{2} G_{7 / 2}$ & $1.24(14)$ & $3.95(13)^{a}$ \\
\hline $2 p^{5} 3 d 4 f$ & ${ }^{2} G_{9 / 2}$ & $1.08(13)$ & $1.77(13)^{a}$ \\
\hline \multicolumn{4}{|c|}{ Mg-like $\mathrm{W}\left(\mathrm{W}^{62+}\right)$} \\
\hline \multicolumn{2}{|c|}{ level } & this work & other works \\
\hline $4 p 4 f$ & ${ }^{3} D_{1}$ & $1.94(11)$ & $0.38(11)^{a}, 0.53(11)^{b}$ \\
\hline $4 f^{2}$ & ${ }^{3} F_{2}$ & $3.64(11)$ & $8.90(11)^{a}, 8.44(11)^{b}$ \\
\hline $4 f^{2}$ & ${ }^{3} P_{0}$ & $6.17(12)$ & $2.42(12)^{a}, 3.92(12)^{b}$ \\
\hline $4 f^{2}$ & ${ }^{3} P_{2}^{*}$ & $6.17(11)$ & $12.76(11)^{a}, 12.32(11)^{b}$ \\
\hline $4 f^{2}$ & ${ }^{3} P_{2}^{*}$ & $6.45(12)$ & $1.75(12)^{a}, 1.59(12)^{b}$ \\
\hline $4 f^{2}$ & ${ }^{1} S_{0}$ & $2.62(13)$ & $1.05(13)^{a}, 1.68(13)^{b}$ \\
\hline $4 d 4 f$ & ${ }^{3} F_{2}$ & $1.26(12)$ & $0.75(12)^{a}, 0.65(12)^{b}$ \\
\hline $4 d 4 f$ & ${ }^{3} P_{2}$ & $1.81(11)$ & $1.74(11)^{a}, 1.23(11)^{b}$ \\
\hline $4 d 4 f$ & ${ }^{3} D_{2}$ & $4.46(11)$ & $3.12(11)^{a}, 1.40(11)^{b}$ \\
\hline $4 f^{2}$ & ${ }^{3} H_{6}$ & $1.97(13)$ & $3.27(13)^{a}$ \\
\hline $4 d 5 g$ & ${ }^{1} I_{6}$ & $3.69(13)$ & $2.64(13)^{a}$ \\
\hline $4 f 5 f$ & ${ }^{3} H_{4}$ & $8.68(12)$ & $6.28(12)^{a}$ \\
\hline $4 d 4 f$ & ${ }^{1} H_{5}$ & $8.85(13)$ & $3.05(13)^{a}$ \\
\hline $4 f 5 d$ & ${ }^{3} G_{5}$ & $2.03(13)$ & $2.50(13)^{a}$ \\
\hline $4 s 5 p$ & ${ }^{3} P_{2}$ & $3.12(13)$ & $1.63(13)^{a}$ \\
\hline $4 d 4 f$ & ${ }^{3} H_{5}$ & $1.46(13)$ & $6.08(12)^{a}$ \\
\hline $4 p 5 g$ & ${ }^{1} \mathrm{~F}_{3}$ & $3.79(12)$ & $2.65(12)^{a}$ \\
\hline $4 s 5 f$ & ${ }^{3} \mathrm{~F}_{3}$ & $3.89(13)$ & $2.28(13)^{a}$ \\
\hline $4 f 5 s$ & ${ }^{3} \mathrm{~F}_{3}$ & $2.26(13)$ & $1.39(13)^{a}$ \\
\hline $4 p 6 g$ & ${ }^{1} \mathrm{~F}_{3}$ & $2.13(12)$ & $2.05(12)^{a}$ \\
\hline $4 f 5 d$ & ${ }^{3} \mathrm{D}_{3}$ & $6.27(12)$ & $6.55(12)^{a}$ \\
\hline $4 f 6 d$ & ${ }^{3} \mathrm{H}_{5}$ & $9.87(12)$ & $7.04(12)^{a}$ \\
\hline $4 s 5 d$ & ${ }^{3} \mathrm{D}_{2}$ & $3.71(13)$ & $2.62(13)^{a}$ \\
\hline $4 p 5 p$ & ${ }^{3} \mathrm{D}_{2}$ & $4.55(12)$ & $9.31(12)^{a}$ \\
\hline $4 s 6 d$ & ${ }^{3} \mathrm{D}_{3}$ & $1.98(13)$ & $1.40(13)^{a}$ \\
\hline $4 f 6 f$ & ${ }^{3} \mathrm{H}_{4}$ & $4.65(12)$ & $4.09(12)^{a}$ \\
\hline
\end{tabular}


TABLE II (cont.)

\begin{tabular}{lc|cc}
\hline \hline $4 s 5 g$ & ${ }^{3} \mathrm{G}_{5}$ & $9.47(12)$ & $4.50(12)^{a}$ \\
$2 p^{5} 3 s 3 p^{2}$ & ${ }^{3} D_{1}$ & $3.17(12)$ & $1.06(13)^{a}, 4.40(12)^{b}$ \\
$2 p^{5} 3 p^{2} 3 d$ & ${ }^{1} P_{1}$ & $5.94(12)$ & $4.13(12)^{a}, 2.39(12)^{b}$ \\
$2 p^{5} 3 s 3 p^{2}$ & ${ }^{5} P_{1}$ & $4.64(11)$ & $1.55(12)^{a}, 6.63(11)^{b}$ \\
$2 p^{5} 3 s^{2} 3 d$ & ${ }^{3} P_{0}$ & $1.62(14)$ & $1.45(14)^{a}, 3.02(14)^{b}$ \\
$2 p^{5} 3 s 3 p^{2}$ & ${ }^{3} P_{0}^{*}$ & $4.59(14)$ & $2.40(14)^{a}, 4.14(14)^{b}$ \\
$2 p^{5} 3 s 3 p^{2}$ & ${ }^{3} P_{0}^{*}$ & $1.45(12)$ & $8.72(11)^{a}, 1.84(12)^{b}$ \\
$2 p^{5} 3 s^{2} 3 d$ & ${ }^{1} D_{2}$ & $5.45(11)$ & $7.20(11)^{a}, 5.70(11)^{b}$ \\
$2 p^{5} 3 s 3 p^{2}$ & ${ }^{1} S_{0}$ & $5.67(12)$ & $1.28(12)^{a}, 1.95(12)^{b}$ \\
$2 p^{5} 3 p^{2} 3 d$ & ${ }^{5} D_{0}^{*}$ & $1.05(11)$ & $1.15(11)^{a}, 1.60(11)^{b}$ \\
$2 p^{5} 3 p^{2} 3 d$ & ${ }^{5} D_{0}^{*}$ & $1.29(12)$ & $3.75(12)^{a}, 1.73(12)^{b}$ \\
$2 p^{5} 3 p^{2} 3 d$ & ${ }^{3} P_{0}^{*}$ & $1.60(12)$ & $1.11(12)^{a}, 1.70(12)^{b}$ \\
$2 p^{5} 3 p^{2} 3 d$ & ${ }^{3} P_{0}^{*}$ & $7.34(10)$ & $4.71(10)^{a}, 4.18(10)^{b}$ \\
$2 p^{5} 3 p^{2} 3 d$ & ${ }^{3} P_{0}^{*}$ & $14.8(12)$ & $9.26(12)^{a}, 7.01(12)^{b}$ \\
$2 p^{5} 3 p^{2} 3 d$ & ${ }^{3} P_{0}^{*}$ & $2.71(11)$ & $2.51(11)^{a}, 3.02(11)^{b}$ \\
$2 p^{5} 3 p^{2} 3 d$ & ${ }^{3} D_{1}$ & $9.08(09)$ & $9.40(09)^{a}, 2.69(10)^{b}$ \\
$2 p^{5} 3 s 3 d^{2}$ & ${ }^{3} D_{2}$ & $6.45(13)$ & $6.52(13)^{a}, 4.82(13)^{b}$ \\
$2 p^{5} 3 s 3 p^{2}$ & ${ }^{5} D_{0}$ & $1.42(14)$ & $1.26(14)^{a}, 2.29(14)^{b}$ \\
${ }^{a} \mathrm{COWAN}$ code, ${ }^{b}$ HULLAC code in Refs. $[14,15]$
\end{tabular}

QED contributions include vacuum polarization and self-energy contributions to level energies. The finitenucleus effect is taken into account by assuming an extended Fermi distribution for the nucleus. The Breit interaction includes magnetic interaction between the electrons and retardation effects of the electron-electron interaction. Both of the Breit and QED contributions are treated as perturbation. Orbitals are fixed, but the mixing coefficients are calculated by diagonalizing the modified Hamiltonian. The correlation effects are taken into account using CI method.

In Table II and Table III, we have listed the autoionization rates and compared with other available works in $[14,15]$ for Na- and Mg-like tungsten, and Na- and Mg-like gold, respectively. Autoionizing levels above the threshold $1 s^{2} 2 s^{2} 2 p^{6}$ for Na-like ions and $1 s^{2} 2 s^{2} 2 p^{6} 3 s$ for Mg-like ions are considered. The autoionization results obtained are too large. We have obtained 539 transitions for $\mathrm{W}^{63+}, 1597$ transitions for $\mathrm{W}^{62+}$ from the selected configurations for tungsten ions to the $2 p^{6} 3 \mathrm{snl}$ autoionizing states and 163 transitions for $\mathrm{Au}^{68+}$ and 426 transitions for $\mathrm{Au}^{67+}$ from the selected configurations for gold ions to the $2 p^{6} n l$ autoionizing states. For this reason, we have here presented just the results which have comparing values for tungsten ions in Table II. Although there are few data for tungsten ions in available literature, there are no data about autoionization results for gold. In the tables, the core of $1 s^{2} 2 s^{2}$ or $1 s^{2} 2 s^{2} 2 p^{6}$ is omitted and the number in brackets represents the power of 10. It is seen in Table II that there is an agreement for Na- and Mg-like tungsten when comparing with [14] and [15], respectively, except some levels. For Mg-like tungsten, some levels have been occurred twice either this work or other works. We have assigned these levels with the superscript ${ }^{*}$. It is seen that there are also disagreement between the results obtained from COWAN
TABLE III

Autoionization rates $\left(A_{a}\right.$ in $\left.\mathrm{s}^{-1}\right)$ for $\mathrm{Na}$ - and $\mathrm{Mg}$-like gold ions. Numbers in brackets represent powers of 10 .

\begin{tabular}{|c|c|c|c|c|}
\hline \multicolumn{3}{|c|}{ Na-like $\mathrm{Au}\left(\mathrm{Au}^{68+}\right)$} & \multicolumn{2}{|c|}{ Mg-like $\mathrm{Au}\left(\mathrm{Au}^{67+}\right)$} \\
\hline \multicolumn{2}{|c|}{ level } & \multirow{2}{*}{$\begin{array}{c}\text { this work } \\
1.11(13)\end{array}$} & level & \multirow{2}{*}{$\frac{\text { this work }}{6.35(13)}$} \\
\hline $2 p^{5} 3 p 3 d$ & ${ }^{4} G_{9 / 2}$ & & $4 d 4 f \quad{ }^{1} H_{5}$ & \\
\hline $2 p^{5} 3 p 3 d$ & ${ }^{2} G_{9 / 2}$ & $1.28(13)$ & $4 s 5 p \quad{ }^{1} P_{1}$ & $5.56(13)$ \\
\hline $2 p^{5} 3 p 3 d$ & ${ }^{4} F_{9 / 2}$ & $1.47(13)$ & $4 s 5 f \quad{ }^{1} F_{3}$ & $5.15(13)$ \\
\hline $2 p^{5} 3 d^{2}$ & ${ }^{2} G_{7 / 2}$ & $1.57(13)$ & $4 f^{2}$ & $4.44(13)$ \\
\hline $2 p^{5} 3 d^{2}$ & ${ }^{4} F_{7 / 2}$ & $4.14(13)$ & $4 f^{2}$ & $3.94(13)$ \\
\hline $2 p^{5} 3 d^{2}$ & ${ }^{2} F_{7 / 2}$ & $4.07(14)$ & $4 s 5 d \quad{ }^{3} D_{1}$ & $3.92(13)$ \\
\hline $2 p^{5} 3 d^{2}$ & ${ }^{4} D_{7 / 2}$ & $3.60(13)$ & $4 s 5 d \quad{ }^{3} D_{3}$ & $3.61(13)$ \\
\hline $2 p^{5} 3 p 3 d$ & ${ }^{2} F_{5 / 2}$ & $1.49(14)$ & $4 f 5 s \quad{ }^{1} F_{3}$ & $3.27(13)$ \\
\hline $2 p^{5} 3 p 3 d$ & ${ }^{4} D_{5 / 2}$ & $1.71(14)$ & $4 s 5 p \quad{ }^{3} P_{2}$ & $3.01(13)$ \\
\hline $2 p^{5} 3 p 3 d$ & ${ }^{2} D_{5 / 2}$ & $1.96(14)$ & $4 s 5 p \quad{ }^{3} P_{0}$ & $2.88(13)$ \\
\hline $2 p^{5} 3 d^{2}$ & ${ }^{2} F_{5 / 2}$ & $8.71(14)$ & $4 s 5 f \quad{ }^{3} F_{3}$ & $2.73(13)$ \\
\hline $2 p^{5} 3 d^{2}$ & ${ }^{4} D_{5 / 2}$ & $1.20(13)$ & $4 s 5 d \quad{ }^{3} D_{2}$ & $2.63(13)$ \\
\hline $2 p^{5} 3 d^{2}$ & ${ }^{2} D_{5 / 2}$ & $6.48(13)$ & $4 s 6 d \quad{ }^{1} D_{2}$ & $2.58(13)$ \\
\hline $2 p^{5} 3 p 3 d$ & ${ }^{4} F_{3 / 2}$ & $7.43(13)$ & $4 f^{2}$ & $2.51(13)$ \\
\hline $2 p^{5} 3 p 3 d$ & ${ }^{2} D_{3 / 2}$ & $3.85(14)$ & $4 s 5 f \quad{ }^{3} F_{2}$ & $2.43(13)$ \\
\hline $2 p^{5} 3 p 3 d$ & ${ }^{2} P_{3 / 2}$ & $6.83(13)$ & $4 s 5 p \quad{ }^{3} P_{1}$ & $2.29(13)$ \\
\hline $2 p^{5} 3 s 3 d$ & ${ }^{4} F_{3 / 2}$ & $1.09(14)$ & $4 s 5 f \quad{ }^{3} F_{4}$ & $2.28(13)$ \\
\hline $2 p^{5} 3 s 3 d$ & ${ }^{4} D_{3 / 2}$ & $2.83(13)$ & $4 f^{2}$ & $2.06(13)$ \\
\hline $2 p^{5} 3 p^{2}$ & ${ }^{2} D_{3 / 2}$ & $1.44(14)$ & $4 s 6 d \quad{ }^{3} D_{1}$ & $2.03(13)$ \\
\hline $2 p^{5} 3 s 3 d$ & ${ }^{2} P_{3 / 2}$ & $1.24(13)$ & $4 s 6 d \quad{ }^{3} D_{3}$ & $2.03(13)$ \\
\hline $2 p^{5} 3 p^{2}$ & ${ }^{2} P_{3 / 2}$ & $4.36(14)$ & $4 f 5 f \quad{ }^{1} G_{4}$ & $2.00(13)$ \\
\hline $2 p^{5} 3 d^{2}$ & ${ }^{2} P_{3 / 2}$ & $8.63(13)$ & $4 d 4 f \quad{ }^{3} H_{5}$ & $1.93(13)$ \\
\hline $2 p^{5} 3 p 3 d$ & ${ }^{4} D_{1 / 2}$ & $2.04(13)$ & $4 f 5 f$ & $1.87(13)$ \\
\hline $2 p^{5} 3 s 3 p$ & ${ }^{4} P_{1 / 2}$ & $2.10(14)$ & $4 s 5 d \quad{ }^{1} D_{2}$ & $1.65(13)$ \\
\hline $2 p^{5} 3 s 3 p$ & ${ }^{2} P_{1 / 2}$ & $2.88(13)$ & $4 s 6 d$ & $1.41(13)$ \\
\hline $2 p^{5} 3 p 3 d$ & ${ }^{2} P_{1 / 2}$ & $2.83(13)$ & $4 s 5 g$ & $1.38(13)$ \\
\hline $2 p^{5} 3 s 3 p$ & ${ }^{2} S_{1 / 2}$ & $4.43(14)$ & $4 d 5 g$ & $1.29(13)$ \\
\hline $2 p^{5} 3 p 3 d$ & ${ }^{2} S_{1 / 2}$ & $9.20(13)$ & $4 s 5 g$ & $1.03(13)$ \\
\hline $2 p^{5} 3 p^{2}$ & ${ }^{4} D_{1 / 2}$ & $1.84(14)$ & $4 f 6 d \quad{ }^{3} H_{5}$ & $1.03(13)$ \\
\hline $2 p^{5} 3 d^{2}$ & ${ }^{4} D_{1 / 2}$ & $3.79(13)$ & & \\
\hline $2 p^{5} 3 s 3 d$ & ${ }^{4} P_{1 / 2}$ & $1.03(13)$ & & \\
\hline $2 p^{5} 3 s 3 d$ & ${ }^{2} P_{1 / 2}$ & $1.27(14)$ & & \\
\hline $2 p^{5} 3 p^{2}$ & ${ }^{2} P_{1 / 2}$ & $4.59(14)$ & & \\
\hline $2 p^{5} 3 d^{2}$ & ${ }^{2} P_{1 / 2}$ & $9.07(13)$ & & \\
\hline
\end{tabular}

and HULLAC codes in [15] for some levels of Mg-like tungsten given in last column in Table II. We can not completely explain these differences. We think that the codes including various relativistic and correlation contributions respond from this case. Of course it is clear that our results, and other results, for these ions, need experimental and observation data.

\section{Conclusion}

Autoionization data of highly ionized tungsten and gold ions are only available for a limited number in the literature. We have here reported new data for $\mathrm{Na}$ - and Mg-like gold ions. Although our results need other computational and experimental works for comparing, we hope that these results obtained from AUTOSTRUCTURE code will be also useful for next works, because the 
autoionization data of highly ionized atoms are very important in plasma physics, astrophysics, ultracold atomic physics, surface science and chemistry (the Auger spectroscopy), laser isotope separation, and development of new lasers.

\section{Acknowledgments}

The authors are very grateful to the anonymous reviewer for stimulating comments and valuable suggestions, which resulted in improving of the paper. This work was supported by Research Fund of the Sakarya University (project No. 2013-50-02-013).

\section{References}

[1] E. Arimondo, C.W. Clark, W.C. Martin, Rev. Mod. Phys. 82, 1947 (2010).

[2] J. Bokor, R.R. Freeman, W.E. Cooke, Phys. Rev. Lett. 48, 1242 (1982).

[3] S.E. Harris, Phys. Rev. Lett. 62, 1033 (1989).

[4] P.T. Greenland, Contemp. Phys. 31, 405 (1990).

[5] A. Lyras, H. Bauchau, Phys. Rev. A 60, 4781 (1999).

[6] A. Apalategui, B.S. Mecking, P. Lambropoulos, Laser Phys. 9, 773 (1999).

[7] E. Paspalakis, N.J. Kylstra, P.L. Knight, Phys. Rev. A 60, 642 (1999).

[8] Y.F. Bai, H. Guo, H. Sun, D.G. Han, C. Liu, X.Z. Chen, Phys. Rev. A 69, 043814 (2004).

[9] T. Köhler, K. Gral, P.S. Julienne, Rev. Mod. Phys. 78, 1311 (2006).

[10] Y. Ohtsuki, Y. Fujimura, Chem. Phys. 338, 285 (2007).

[11] Ş. Erkoç, T. Uzer, Atomic and Molecular Physics, World Sci., Singapore 1996.

[12] S. Hamasha, R. Alshaiub, Phys. Scr. 86, 065302 (2012).

[13] F. Hu, C. Wang, J. Yang, G. Jiang, L. Hao, Phys. Scr. 84, 015302 (2011).

[14] U.I. Safronova, A.S. Safronova P. Beiersdorfer, At. Data Nucl. Data Tables 95, 751 (2009).

[15] U.I. Safronova, A.S. Safronova, P. Beiersdorfer J. Phys. B 42, 165010 (2009).

[16] U.I. Safronova, A.S. Safronova, W.R. Johnson, J. Phys. B 43, 144001 (2010)
[17] U.I. Safronova, A.S. Safronova, P. Beiersdorfer, W.R. Johnson, J. Phys. B 44, 035005 (2011).

[18] U.I. Safronova, A.S. Safronova, Phys. Rev. A 85, 032507 (2012).

[19] U.I. Safronova, A.S. Safronova, Phys. Rev. A 86 , 042510 (2012).

[20] U.I. Safronova, A.S. Safronova, P. Beiersdorfer, J. Phys. B 45, 085001 (2012).

[21] U.I. Safronova, A.S. Safronova, Phys. Rev. A 91, 062507 (2015).

[22] F.C. Meng, C.Y. Chen, Y.S. Wang, Y.M. Zou, J. Quant. Spectrosc. Radiat. Transfer 109, 2000 (2008).

[23] B.W. Li, G. O'Sullivan, Y.B. Fu, C.Z. Dong, Phys. Rev. A 85, 052706 (2012).

[24] M. Li, Y. Fu, M. Su, C. Dong, F. Koike, Plasma Sci. Technol. 16, 182 (2014).

[25] S.P. Preval, N.R. Badnell, M.G. O'Mullane, Phys. Rev. A 93, 042703 (2016).

[26] D.H. Kwon, W. Lee, J. Quant. Spectrosc. Radiat. Transfer 170, 182 (2016).

[27] F.C. Meng, C.Y. Chen, X.H. Shi, Y.S. Wang, Y.M. Zou, M.F. Gu, J. Phys. B 40, 4269 (2007).

[28] J.H. Yang, X.L. Cheng, B.L. Deng, Phys. Scr. 81 , 015304 (2010).

[29] C.P. Ballance, D.C. Griffin, S.D. Loch, N.R. Badnell, J. Phys. B 45, 045001(2012).

[30] G. Xiong, J. Zhang, Z. Hu, N. Nakamura, Y. Li, X. Han, J. Yang, B. Zhang, Phys. Rev. A 88, 042704 (2013).

[31] N.R. Badnell, Comput. Phys. Commun. 182, 1528 (2011).

[32] N.R. Badnell, J. Phys. B 19, 3827 (1986).

[33] N.R. Badnell, J. Phys. B 30, 1 (1997).

[34] W. Eissner, M. Jones, H. Nussbaumer, Comput. Phys. Commun. 8, 270 (1974).

[35] R.D. Cowan, The Theory of Atomic Structure and Spectra, University of California Press, USA 1981.

[36] L. Özdemir, G.G. Konan, S. Kabakçı, Acta Phys. Pol. A 124, 649 (2013).

[37] G.G. Konan, L. Özdemir, G. Ürer, J. Quant. Spectrosc. Radiat. Transfer 145, 110 (2014).

[38] G.G. Konan, L. Özdemir, Chin. J. Phys. 54, 433 (2016).

[39] G.G. Konan, L. Özdemir, Can. J. Phys. 94, 1167 (2016). 